Recepción: 20 / 04 / 2017

Aceptación: 20 / 05 / 2017

Publicación: 15 / 06 / 2017

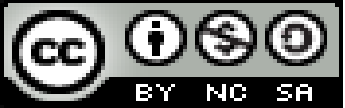

Ciencias Sociales y Políticas

Artículo de Investigación

\title{
Relación de género y su incidencia en la participación de las políticas universitarias en una Universidad en el Ecuador
}

Gender relations and their impact on the participation of university policies in a University in Ecuador

Relações de gênero e seu impacto sobre a participação de políticas universitárias em uma universidade no Equador

Leónidas I. Viejó-Mora ${ }^{\mathrm{I}}$ cleoisraelviejo@yahoo.com

Dennis M. Jiménez-Bonilla II mauri0204@hotmail.com

Víctor S. Cabezas-Pinta ${ }^{\text {III }}$ victor_che1725@hotmail.com

Correspondencia: cleoisraelviejo@yahoo.com

\footnotetext{
I. Licenciado en Ciencias de la Comunicación Social Mención en Periodismo; Docente de la Universidad Estatal de Milagro, Guayas, Ecuador.

II. Magister en Psicología Laboral con Mención en Desarrollo Humano y de la Organización, Psicólogo Organizacional, Docente de la Universidad Estatal de Milagro, Guayas, Ecuador.

III. Licenciado en Ciencias de la Comunicación Social mención en Periodismo; Docente de la Universidad Estatal de Milagro, Guayas, Ecuador.
} 


\title{
Resumen
}

La investigación que se presenta aborda sobre el análisis cuantitativo y cualitativo en el nivel de participación de las mujeres con la participación de los hombres en política universitaria, realizado en la Universidad Estatal de la Ciudad de Milagro, provincias del Guayas de la República del Ecuador.

Para ello se ha considerado importante investigar y analizar, la correlación de la participación de mujeres y hombres en política universitaria antes y después de la aprobación de la constitución del Ecuador, tomando la relación de los años 2007 y 2015.

Palabras claves: Género, Constitución, Estado, Política, participación.

\begin{abstract}
The present research deals with the quantitative and qualitative analysis on the level of participation of women with the participation of men in university politics, carried out at the State University of the City of Milagro, provinces of the Guayas of the Republic of Ecuador.

For this purpose, it has been considered important to investigate and analyze the correlation between the participation of women and men in university politics before and after the approval of the Ecuadorian constitution, taking the relationship of the years 2007 and 2015.
\end{abstract}

Key words: Gender, Constitution, State, Politics, participation.

\section{Resumo}

A pesquisa apresentada endereços na análise quantitativa e qualitativa sobre o nível de participação das mulheres, com a participação de homens em política universitária, realizado pela Universidade Estadual Cidade Milagro, Guayas Província da República do Equador.

Por isso tem sido considerado importante para investigar e analisar a correlação da participação de mulheres e homens na política universitária antes e depois da adopção da Constituição do Equador, tendo a proporção dos anos de 2007 e 2015.

Palavras-chave: Sexo, constituição, estado, política, participação. 


\section{Introducción.}

Desde el año 1830 que se aprobó la primera constitución de la República del Ecuador, hasta el año 2007, la participación de la mujer en la política nacional como en política universitaria, era muy baja, por no decir nada. Esto debido a muchos factores: a) la discriminación (Torres Falcón, 2005) por parte de los hombres en que las mujeres estén en espacios de poder y muchas veces sean quienes lideren. b) El poco apoyo de los padres porque las mujeres estén involucradas en la política. (Ricón, 2015) c) el machismo absoluto de novios u esposos porque las mujeres hagan actividades que según ellos sólo deben hacer los hombres. (Zapata-Martelo \& Ayala-Carrillo, 2014)

Todos estos paradigmas eran trasmitidos $\mathrm{u}$ observados en aquellos tiempos, donde la sociedad era discriminante en la participación femenina. (Rivas, M; Sajardo, A, 2004)

A raíz de la aprobación de la nueva constitución de la república del Ecuador en el 2008, tras haberse llamado a una constituyente en la ciudad de Montecristi provincia de Manabí, se da un cambio de página a toda la estructura republicana del Ecuador y se comienza a establecer como política pública en todas las leyes trasversales que emerge en esta carta magna, la equidad de género (Archendi, N; Tula, M, 2014), en donde se da el mismo nivel, jerarquía y derecho a la mujer, en temas de concursos de merecimientos público y participación electoral.

Indicando la segunda en mención, que ninguna lista de elección popular podrá conformarse sin la alternabilidad de mujeres y hombres.

Desde este momento, el porcentaje de ingreso de las mujeres a las universidades comienza crecer, a su vez el nivel de participación en las curules de política universitaria. 
Si bien es cierto que este crecimiento se ha dado en gran medida en un cincuenta porciento en los cargos de elección popular, donde es el pueblo quien elige, aún falta mucho porque el liderazgo y el compromiso de las mujeres se vea reflejado no sólo en ocupar una curul (cargo o dignidad), sino también se vea reflejado en el apoderamiento de los cambios y políticas sociales. (Minteguiaga, 2012) 


\section{Materiales y métodos.}

La metodología llevada a cabo dentro de la investigación, como primer paso, fue el levantamiento de documentos y el análisis de la participación correlacionada de género antes y después de la aprobación de la constitución. (Martínez, 2009) Para luego conllevar a la realización de entrevista pública dirigida a 1.000 estudiantes universitarios, con el propósito de conocer su opinión, si consideran que las mujeres están en la capacidad de asumir el liderazgo dentro de la Universidad y su incidencia activa en la política universitaria.

Ubicando como referencia una pregunta de la encuesta. En la cual el 52\% consideran que las mujeres si están en los actuales momentos en la capacidad de asumir el liderazgo político en la Universidad Estatal de Milagro.

De la misma manera, se encuestó a 100 padres de familias, con el propósito de conocer si estarían dispuestos en apoyar que su hija participe en política universitaria. Encontrado como resultado que el $52 \%$ de los padres de familia indicaban que no, mientras que un $48 \%$ indican que si lo harían.

\section{Resultados.}

\begin{tabular}{|l|l|l|l|}
\hline Modalidad & Mujeres & Hombre & Sub total \\
\hline Presencial & 2.249 & 1.110 & 3.359 \\
\hline Semipresencial & 670 & 469 & 1139 \\
\hline & & Total $=$ & 4498 \\
\hline
\end{tabular}

Cuadro 1

Estudiantes matriculados en la Universidad Estatal de Milagro segundo semestre año 2007 


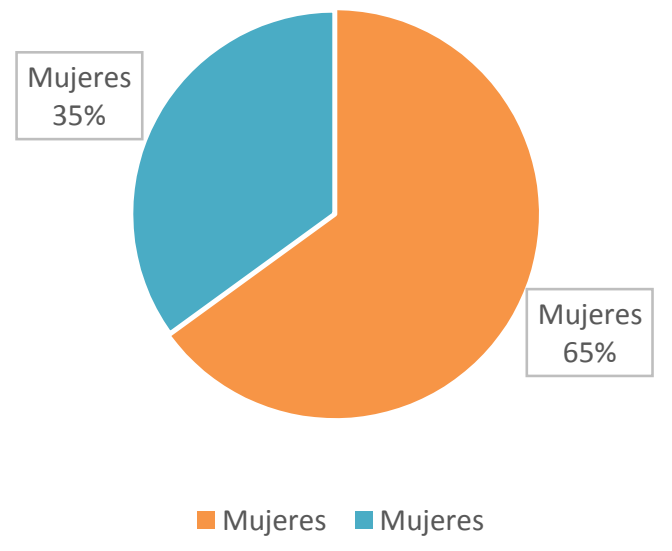

\section{Gráfico 1}

Estudiantes matriculados en la Universidad Estatal de Milagro año 2007

\section{ANÁLISIS}

De acuerdo a la documentación facilitada por la secretaría general de la Universidad Estatal de Milagro, los estudiantes matriculados en el año 2007 son 4.498, de los cuales el 65\% son mujeres y el $35 \%$ hombres

\begin{tabular}{|c|c|c|c|c|}
\hline Facultad & Dignidad & $\begin{array}{l}\text { Miembros a } \\
\text { integrar }\end{array}$ & Mujeres & Hombres \\
\hline $\begin{array}{l}\text { Ciencias de la } \\
\text { Educación }\end{array}$ & Asociación Escuela & 5 & 2 & 3 \\
\hline comunicación & & & & \\
\hline $\begin{array}{l}\text { Ciencias de la } \\
\text { Ingeniería }\end{array}$ & Asociación Escuela & 5 & 1 & 4 \\
\hline $\begin{array}{l}\text { Ciencias } \\
\text { Administrativas } \\
\text { y comerciales }\end{array}$ & Asociación Escuela & 5 & 2 & 3 \\
\hline $\begin{array}{l}\text { Ciencias de la } \\
\text { Salud }\end{array}$ & Asociación Escuela & 5 & 3 & 2 \\
\hline Semipresencial & Asociación Escuela & 5 & 3 & 2 \\
\hline
\end{tabular}




\begin{tabular}{|c|c|c|c|c|}
\hline \multicolumn{5}{|l|}{ y a distancia } \\
\hline Universidad & $\begin{array}{ll}\text { Federación } & \text { de } \\
\text { Estudiantes } & \\
\text { Universitarios } & \text { de } \\
\text { Milagro } & \end{array}$ & 5 & 2 & 3 \\
\hline Universidad & $\begin{array}{ll}\text { Liga } & \text { Deportiva } \\
\text { Universitaria } & \end{array}$ & 5 & 1 & 4 \\
\hline \multirow[t]{2}{*}{ Universidad } & $\begin{array}{ll}\text { Asociación } & \text { Femenina } \\
\text { Universitaria } & \end{array}$ & & 5 & 0 \\
\hline & Total & 35 & 19 & 21 \\
\hline
\end{tabular}

\section{Cuadro 2}

\section{Estadística de participación en política universitarios de mujeres y hombre en el año 2007}

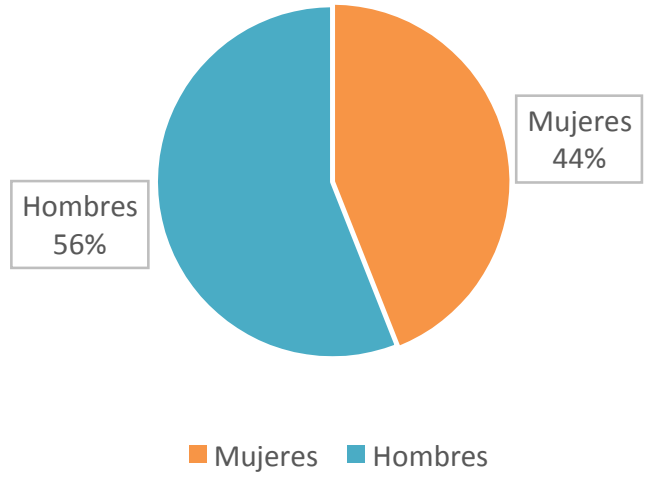

\section{Gráfico 2}

Correlación de género de participación en política universitaria año 2007

\section{ANÁLISIS}

Dentro de los documentos recopilados de la Federación de Estudiantes Universitarios del Ecuador Filial Milagro, podemos constatar que la correlación de género en política universitaria en 
el año 2007, demuestra que la participación de los hombres está ubicada en un 56\%, mientras que la participación de las mujeres está en 44\%.

Esto es en lo que respecta al registro de curules en cargos estudiantiles en participación electoral, mas no en nivel de liderazgo y apoderamiento social

\begin{tabular}{|l|l|l|l|}
\hline Modalidad & Mujeres & Hombre & Sub total \\
\hline Presencial & 2.817 & 1.337 & 3.744 \\
\hline Semipresencial & 300 & 110 & 410 \\
\hline & & Total $=$ & 4.154 \\
\hline
\end{tabular}

\section{Cuadro 3}

Estudiantes matriculados en la Universidad Estatal de Milagro segundo semestre año 2015

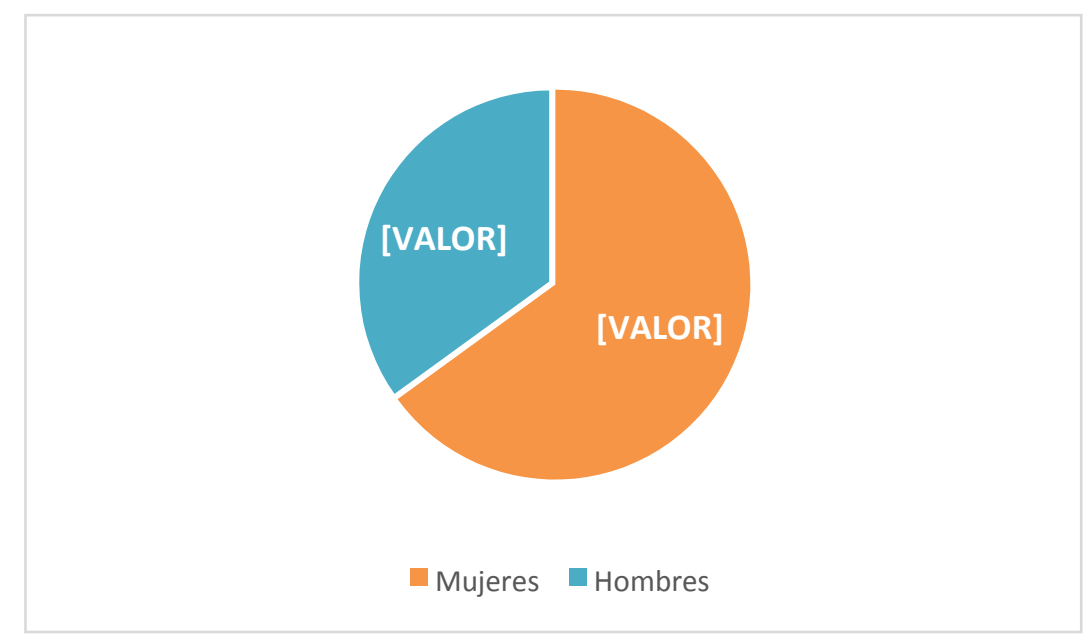

Gráfico 3

Estudiantes matriculados en la Universidad Estatal de Milagro año 2015 


\section{ANÁLISIS}

De acuerdo al registro de estudiantes matriculados en la Universidad correspondiente al año 2015, podemos constatar que hay 4.154 estudiantes, de los cuales el $65 \%$ son mujeres, mientras que el $35 \%$ son hombres.

\begin{tabular}{|c|c|c|c|c|}
\hline $\begin{array}{l}\text { Universidad } \\
\text { Facultad }\end{array}$ & Dignidad & $\begin{array}{l}\text { Miembros a } \\
\text { integrar }\end{array}$ & Mujeres & Hombres \\
\hline $\begin{array}{lr}\text { Ciencias de } & \text { la } \\
\text { Educación } & y \\
\text { comunicación } & \end{array}$ & $\begin{array}{l}\text { Asociación } \\
\text { Escuela }\end{array}$ & 14 & 7 & 7 \\
\hline $\begin{array}{l}\text { Ciencias } \\
\text { Ingeniería }\end{array}$ & $\begin{array}{l}\text { Asociación } \\
\text { Escuela }\end{array}$ & 14 & 7 & 7 \\
\hline $\begin{array}{l}\text { Ciencias } \\
\text { Administrativas } \quad \text { y } \\
\text { comerciales }\end{array}$ & $\begin{array}{l}\text { Asociación } \\
\text { Escuela }\end{array}$ & 14 & 7 & 7 \\
\hline $\begin{array}{lll}\text { Ciencias } & \text { de } & \text { la } \\
\text { Salud } & & \end{array}$ & $\begin{array}{l}\text { Asociación } \\
\text { Escuela }\end{array}$ & 14 & 7 & 7 \\
\hline $\begin{array}{l}\text { Semipresencial y a } \\
\text { distancia }\end{array}$ & $\begin{array}{l}\text { Asociación } \\
\text { Escuela }\end{array}$ & 14 & 7 & 7 \\
\hline Universidad & $\begin{array}{l}\text { Federación de } \\
\text { Estudiantes } \\
\text { Universitarios } \\
\text { de Milagro }\end{array}$ & 14 & 7 & 7 \\
\hline Universidad & $\begin{array}{l}\text { Liga } \\
\text { Deportiva } \\
\text { Universitaria }\end{array}$ & 14 & 7 & 7 \\
\hline Universidad & $\begin{array}{l}\text { Asociación } \\
\text { Femenina } \\
\text { Universitaria }\end{array}$ & 14 & 14 & 0 \\
\hline & Total & 112 & 63 & 49 \\
\hline
\end{tabular}

Estadística de participación en política universitarios de mujeres y hombre en el 2015 Cuadro 4 


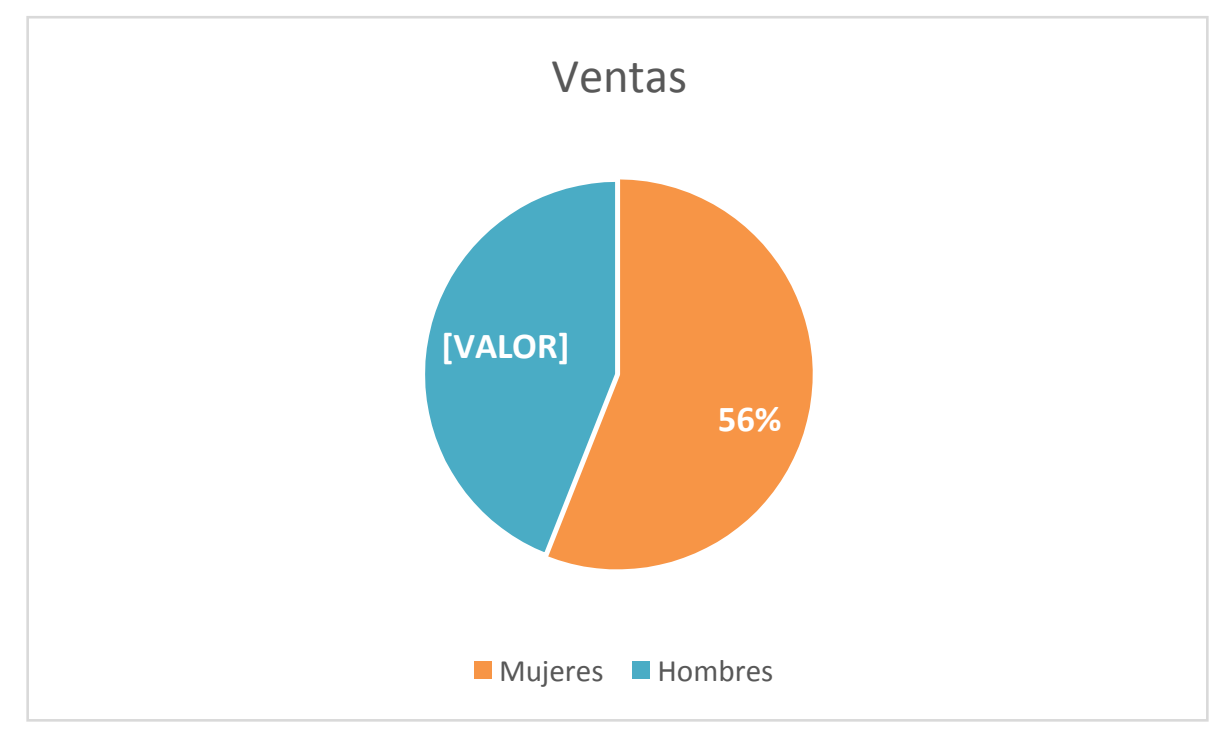

\section{Gráfico 4 \\ Registro de correlación de género en la participación de política universitaria}

\section{ANÁLISIS}

En el año 2015, a raíz de la aprobación de la constitución de la república del Ecuador, que obliga mandatoriamente se cumpla la equidad de género en política electoral, se demuestra que el $56 \%$ de la participación es de las mujeres, mientras que el $44 \%$ es de hombres.

Por lógica, si la constitución me indica que debe existir equidad de género en las elecciones electorales, por qué no cuadra el porcentaje del 50 - 50.

Cabe indicar que dentro de la estructura de los gremios universitarios que agrupa la Federación de Estudiantes Universitarios, existe la Asociación Femenina Universitaria que sólo está constituida por mujeres de acuerdo a la estructura orgánica del gremio. 


\section{ENCUESTAS}

\begin{tabular}{|l|l|l|}
\hline ITEMS & CANTIDAD & PORCENTAJE \\
\hline SI & 52 & $52 \%$ \\
\hline NO & 48 & $48 \%$ \\
\hline TOTAL & 1000 & $100 \%$ \\
\hline
\end{tabular}

¿Considera usted que las mujeres están en la capacidad de asumir liderazgo dentro de la Universidad y el País?

Cuadro 5

\section{Encuesta a universitarios \\ $48 \%$ \\ $52 \%$ \\ $\square \mathrm{Si}$ NO}

Grafica 5

\begin{tabular}{|l|l|l|}
\hline ITEMS & CANTIDAD & PORCENTAJE \\
\hline SI & 38 & $48 \%$ \\
\hline NO & 62 & $52 \%$ \\
\hline TOTAL & 100 & $100 \%$ \\
\hline
\end{tabular}

¿Apoyaría usted a que su hija participe en política Universitaria?

\section{Cuadro 6}




\section{Encuesta a padres de familia}

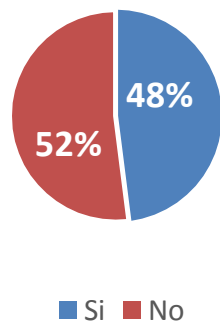

Grafica 6

\section{Conclusiones.}

De acuerdo a la investigación cuantitativa y descriptiva realizado en el año 2007 y 2015, se puede evidenciar el siguiente resultado:

Los estudiantes universitarios matriculados en el año $2007,65 \%$ son mujeres y $35 \%$ hombres. Pero en el nivel de participación política universitaria cambia este resultado, demostrando que $56 \%$ de los hombres ocupan cargos en las dignidades que agrupa la Federación de Estudiantes Universitarios del Ecuador filial Milagro, mientras que 44\% son mujeres. Este resultado es antes de la aprobación de la constitución de la República del Ecuador que se efectuó a través de referéndum en el año 2008.

En el año 2015 se registras un porcentaje de estudiantes universitarios matriculados de $65 \%$ mujeres y 35\% hombres. Mientras que en la Correlación de participación universitaria en los diferentes gremios de la Federación de Estudiantes Universitarios de Milagro, las mujeres tienen un $56 \%$ mientras que los hombres $44 \%$. En este año las curules de las mujeres aumentan más que las de los hombres. 
Si bien es cierto a raíz de la aprobación de la nueva constitución del Ecuador, el nivel de curules en la política universitaria a nivel femenina aumentó, aún no ha crecido el nivel de apoderamiento de la participación activa de las mujeres. La cual evidencia poca libertad de los padres (mamá y papá) que sus hijas estén activando temas políticos, porque se adentran al cuidado de ellas limitando su accionar, donde a través del subconsciente generan un pensamiento patriarcal.

Por otro lado se registra lideresas universitarias que son esposa y madre, donde su campo de acción es limitado por sus esposos por cuanto como es mujer, “deben dedicarse a los quehaceres de familia y no puede descuidar su rol de madre y esposa”.

De acuerdo a una encuesta realizada a padres de familias de diferentes lideresas universitarias, el $48 \%$ de los padres respondieron que si apoyan que sus hijas estén activando en política universitaria, mientras que un $52 \%$ no quiere ver a su hija haciendo activismo universitario.

Constatando que aún hace falta dar un cambio estructural en la forma de pensar de los padres en la participación activa y libre de las mujeres. De nada sirve incrementar los niveles de participación y política pública, si existe restricción de que las mujeres puedan hacerla.

\section{Bibliografía.}

Archendi, N; Tula, M. (2014). Cambios Normatios y equidad de género de las cuotas a la paridad en América Latina: Los casos de Bolivia y Ecuador. America Latina Hoy, 60(1), 47-68.

Martínez, R. (2009). El proyecto de Constitución de Ecuador, ejemplo del nuevo constitucionalismo latinoamericano. IUS. Revista del Instituto de Ciencias Jurídicas de Puebla A.C., 23(1), 264-274.

Minteguiaga, A. (2012). Politicas y politicas sociales en el Ecuador recinete: Dificultades asociadas a la salida de ciclo neoliberal. Revista de Ciencias Sociales, 135(1), 45-58.

Ricón, L. (2015). Mujer y poder político en Latinoamérica: Una reflexión desde el siglo XXI. Utopía y Praxis Latinoamericana, 20(68), 65-78.

Rivas, M; Sajardo, A. (2004). La desigual participación de hombres y mujeres en la economía social: teorías explicativas. CIRIEC-España, Revista de Economía, 1(50), 77-103. 
Torres Falcón, M. (2005). Género y discriminación. El Cotidiano, 134(1), 71-77.

Zapata-Martelo, E., \& Ayala-Carrillo, M. (2014). POLÍTICAS DE EQUIDAD DE GÉNERO: EDUCACIÓN PARA UNA ESCUELA LIBRE DE VIOLENCIA. Ra Ximhai, 10(7), 1-21. 